\title{
Transfusion-Related Hypocalcemia After Trauma
}

\author{
Saskya Byerly ${ }^{1}$ (D) Kenji Inaba $^{2} \cdot$ Subarna Biswas $^{2} \cdot$ Eugene Wang $^{2} \cdot$ Monica D. Wong $^{2}$. \\ Ira Shulman ${ }^{2}$ Elizabeth Benjamin ${ }^{2} \cdot$ Lydia Lam $^{2} \cdot$ Demetrios Demetriades $^{2}$
}

Accepted: 17 July 2020 / Published online: 30 July 2020

(C) Société Internationale de Chirurgie 2020

\begin{abstract}
Background Hypocalcemia is cited as a complication of massive transfusion. However, this is not well studied as a primary outcome in trauma patients. Our primary outcome was to determine if transfusion of packed red blood cells (pRBC) was an independent predictor of severe hypocalcemia (ionized calcium $\leq 3.6 \mathrm{mg} / \mathrm{dL}$ ).

Methods Retrospective, single-center study (01/2004-12/2014) including all trauma patients $\geq 18$ yo presenting to the ED with an ionized calcium (iCa) level drawn. Variables extracted included demographics, interventions, outcomes, and iCa. Regression models identified independent risk factors for severe hypocalcemia (SH).

Results Seven thousand four hundred and thirty-one included subjects, $716(9.8 \%)$ developed SH within $48 \mathrm{~h}$ of admission. Median age: 39 (Range: 18-102), systolic blood pressure: 131 (IQR: 114-150), median Glasgow Coma Scale (GCS): 15 (IQR: 10-15), Injury Severity Score (ISS): 14 (IQR: 9-24). SH patients were more likely to have depressed GCS ( 13 vs $15, p<0.0001)$, hypotension $(23.2 \%$ vs $5.1 \%, p<0.0001)$ and tachycardia $(57.0 \%$ vs $41.9 \%$, $p<0.0001)$ compared to non-SH patients. They also had higher emergency operative rate $(71.8 \%$ vs $29 \%$, $p<0.0001)$ and higher blood administration prior to minimum iCa [pRBC: $(8$ vs $0, p<0.0001$ ), FFP: (4 vs 0 , $p<0.0001$ ), platelet: ( 1 vs $0, p<0.0001)$ ]. Multivariable analysis revealed penetrating mechanism (AOR: 1.706 ), increased ISS (AOR: 1.029), and higher pRBC (AOR: 1.343) or FFP administered (AOR: 1.097) were independent predictors of SH. SH was an independent predictor of mortality (AOR: 2.658). Regression analysis identified a significantly higher risk of SH at pRBC + FFP administration of 4 units (AOR: 18.706, AUC:. 897 (0.884-0.909). Conclusion Transfusion of $\mathrm{pRBC}$ is an independent predictor of $\mathrm{SH}$ and is associated with increased mortality. The predicted probability of SH increases as $\mathrm{pRBC}+$ FFP administration increases.
\end{abstract}

This study was presented at the 2016 Annual Meeting of the American College of Surgeons (ACS), Washington, DC, October 16, 2016.

Kenji Inaba

kinaba@med.usc.edu; kinaba@surgery.usc.edu

Saskya Byerly

s.e.byerly@gmail.com; saskya.byerly@jhsmiami.org

Subarna Biswas

subarna.biswas@med.usc.edu

Eugene Wang

ewangwhc@gmail.com
Monica D. Wong

monica.wong@med.usc.edu

Ira Shulman

ishulman@usc.edu

Elizabeth Benjamin

Elizabeth.benjamin@med.usc.edu

Lydia Lam

Lydia.lam@med.usc.edu 


\section{Introduction}

Symptomatic hypocalcemia occurs with decreased levels of circulating ionized calcium. In general, half of the total circulating calcium is ionized with $40 \%$ bound to plasma proteins, primarily albumin, and $10 \%$ to anions such as sulfate, citrate, and phosphate [1]. Total calcium is often used as a surrogate for ionized calcium, adjusting for hypoalbuminemia. The adjusted calcium formula may not adequately reflect ionized calcium concentrations, however, and is not a reliable substitute in critically ill trauma patients [2-6]. While reference ranges vary by institution, cited lower limit of normal ionized calcium ranges between $4 \mathrm{mg} / \mathrm{dL}(1 \mathrm{mmol} / \mathrm{dL})$ and $4.8 \mathrm{mg} / \mathrm{dL}(1.2 \mathrm{mmol} / \mathrm{dL})$ [7] Hypocalcemia as a result of transfusion is posited to occur via the citrate anticoagulant contained in stored blood. The transfused citrate binds ionized calcium in the recipient, and the resultant hypocalcemia is thought to be most prominent after the transfusion of plasma and platelets, due to their high citrate concentration. More than $90 \%$ of the citrate present in an original blood donation bag is found in plasma and platelets, since citrate partitions with plasma and is separated from the pRBCs during component separation [8]. Hypocalcemia is typically transient in standard transfusion because citrate undergoes rapid hepatic metabolism. The healthy adult liver metabolizes 3 grams of citrate per 1 unit of blood administered every 5 min [9]. Massive transfusion may result in a large influx of citrate, amplified by an impairment in citrate metabolism due to hypothermia, hypoperfusion and liver dysfunction [10-12]. Patients with citrate toxicity resulting in hypocalcemia may present with prolonged QT on EKG, tetany, decreased myocardial contractility, refractory hypotension, or arrhythmia $[10,12,13]$. While hypocalcemia is often cited as a sequelae of transfusion of blood products, there are limited studies that examine hypocalcemia and its relationship to blood transfusion as a primary outcome.

The primary objective of this study was to determine if the transfusion of $\mathrm{pRBC}$ is an independent predictor of hypocalcemia in trauma patients. The second objective was to determine if ionized calcium decreased in a dose-

Demetrios Demetriades

demetria@usc.edu

1 Department of Surgery, Division of Trauma and Critical Care, Jackson Memorial Hospital Ryder Trauma Center, University of Miami, Miami, FL, USA

2 Department of Surgery, Division of Trauma and Critical Care Surgery, Keck School of Medicine, LAC+USC Medical Center, University of Southern California, 2051 Marengo Street, Inpatient Tower, C5L100, Los Angeles, CA 90033, USA dependent relationship with transfusion of pRBC. We hypothesized that $\mathrm{pRBC}$ administration was an independent predictor of hypocalcemia.

\section{Methods}

This was a retrospective single-center study including all trauma patients $\geq 18$ yo presenting to the emergency department (ED) that had an ionized calcium level drawn within $48 \mathrm{~h}$ of arrival within a decade.

After Institutional Review Board (IRB) approval was obtained, the Los Angeles County + University of Southern California electronic medical records was queried for all trauma patients with a total calcium or ionized calcium (iCa) drawn within $48 \mathrm{~h}$ of admission (01/2004-12/2014). Our institution reference for ionized calcium is $4.5 \mathrm{mg} / \mathrm{dL}-$ $5.3 \mathrm{~g} / \mathrm{dL}$ with a critical low trigger of $3.6 \mathrm{mg} / \mathrm{dL}$, similar to previously cited articles of symptomatic hypocalcemia. These patients were cross-referenced with the Trauma Registry and all patients less than 18 years were excluded. For all patients included in this study criteria, age, sex, race, mechanism of injury, systolic blood pressure (SBP), heart rate, Glasgow Coma Scale (GCS) score, Injury Severity Score (ISS), AIS by body region, blood product requirements, interventions, discharge capacity, discharge disposition, ventilator days, hospital length of stay (LOS), ICU LOS, and in-hospital mortality were abstracted. All iCa values for each patient and the time of the iCa sample collection were collected and analyzed. First, minimum iCa and last $\mathrm{iCa}$ along with time drawn after admission were calculated. An $\mathrm{iCa} \leq 3.6 \mathrm{mg} / \mathrm{dL}$ was defined as severe hypocalcemia, as previously described in the literature.

Descriptive statistics were calculated for all variables. Independent $t$ test was used for normally distributed continuous data, which were reported as mean $\pm \mathrm{SD}$. MannWhitney $U$ was used for not normally distributed ordinal and continuous data and reported as median (intraquartile range). Pearson's Chi-squared test or Fisher's exact test were used for categorical data and were reported as percentages. Univariate analysis was used to identify variables predictive of hypocalcemia. Independent variables with $p$ values $<0.2$ and variables of known clinical significance were further analyzed with multivariable analysis. Potential predictors were assessed in a backwards stepwise fashion, and retained for statistical significance or for a greater than $15 \%$ change in the severe hypocalcemia effect estimate. Transfused blood products were maintained as continuous variables in the regression model. Multivariable regression was similarly performed for mortality and for the effect of combined pRBC + FFP administration of 4 units on mortality. Potential effect modification was evaluated by testing for significant interactions between variables. 
$P$ values $<0.05$ were considered statistically significant. Area Under the Receiving Operator Characteristic (AUROC) was used to assess discriminative power of the regression model. Adjusted odds ratio (AOR) with 95\% confidence interval (CI) was derived from the logistic regression. Statistical analysis was performed using SPSS for Mac, version 23 (IBM Corporation, Armonk, NY) and $\mathrm{R}$ version 3.2.2 (R Foundation for Statistical Computing, Vienna, Austria).

\section{Results}

Of the 45,996 trauma patients admitted during the 11-year study period, $7341(16.0 \%)$ were adult patients with iCa drawn within the first $48 \mathrm{~h}$. Of these patients meeting inclusion criteria, 716 (9.8\%) developed severe hypocalcemia within $48 \mathrm{~h}$ of admission. The study population was predominantly male (81.2\%), with a median age 39 (Range: 18-102) years, SBP 131 (IQR: 114-150), median GCS 15 (IQR: 10-15), ISS 14 (IQR: 9-25). The most common mechanism of injury was fall $(20.2 \%)$ followed by motor vehicle collision (18.9\%).

Median mininum ionized calcium was $3.1(2.5-3.4)$ in the severe hypocalcemia group and $4.4 \pm(4.1-4.7)$ in the non-severe hypocalcemic group. Severe hypocalcemia patients were more likely to have a depressed GCS 13 (3 vs $15, p<0.0001)$, hypotension $(23.2 \%$ vs $5.1 \%)$ and tachycardia $(57 \%$ vs $41.9 \%, p<0.0001)$ when compared to non-hypocalcemia patients. The Maximum Abbreviated Injury Scale (AIS) for Head, Chest, Abdomen/Pelvis, Extremities, and External were all greater in hypocalcemic patients (Table 1). Severe hypocalcemic patients had higher rates of intubation ( $47.1 \%$ vs $28.4 \%, p<0.0001$ ) and higher emergency operation rates $(71.8 \%$ vs $29.0 \%$, $p<0.0001$ ).

Patients that ultimately developed severe hypocalcemia were administered more blood products prior to minimum iCa drawn [pRBC ( 8 vs $0, p<0.0001$ ), plasma (4 vs 0 , $p<0.0001)$, and platelets ( 0 vs $0, p<0.0001)$ ]. They also received more blood products within $24 \mathrm{~h}$ of admission [24-h pRBC (11 vs $0, p<0.0001$ ), 24-h plasma ( 8 vs 0 , $p<0.0001$ ), 24-h platelet ( 1 vs $0, p<0.0001$ )] as well as during their total hospital length of stay. Overall, $38.8 \%$ of patients received a blood transfusion prior to the iCa nadir with a higher percent of patients in the hypocalcemia group receiving blood $(94.7 \%$ vs $32.9 \%, p<0.0001)$. Overall, $9.1 \%$ of patients received a massive transfusion defined as 10 or more units of pRBC within $24 \mathrm{~h}$ of admission. This was higher in the hypocalcemia group $(59.4 \%$ vs $3.7 \%$, $p<0.0001)$. Mean last ionized calcium within $48 \mathrm{~h}$ of admission was $4.5(4.0-4.8)$ in the severe hypocalcemic group compared to $4.7(4.5-4.8)$ in the non-severe hypocalcemic group $(p<0.001)$ (Table 2$)$.

Only $34.6 \%$ severe hypocalcemic patients went home at discharge compared to $54.1 \%$ of non-severe hypocalcemic patients. Hypocalcemic patients had longer hospital LOS (9 vs 7 days, $p<0.0001)$, more frequently required ICU support $(79.7 \%$ vs $74.2 \%, p<0.0001)$, had a longer ICU LOS ( 4 vs 3 days, $p<0.0001$ ), and had a higher mortality $(37.7 \%$ vs $10.1 \%, p<0.0001)$ (Table 3 ).

Multivariable analysis revealed that independent predictors of severe hypocalcemia were a penetrating mechanism (AOR 1.706), increased ISS (AOR 1.029), higher pRBC administration (AOR 1.343), and higher plasma administration (AOR 1.097) (Table 4). Testing for interactions between variables revealed an interaction between pRBC and platelet and no other significant interactions were found. In this model, AUROC $=0.909(95 \% \mathrm{CI}=$ $0.897-0.921, p<0.0001)$. Independent predictors of mortality were penetrating mechanism (AOR 1.601), higher ISS (AOR 1.120), severe hypocalcemia (AOR 2.658), older age (AOR 1.040), platelet administration (AOR 1.389), and cryoprecipitate administration (AOR 1.058). In this model, AUROC $=0.888(95 \% \quad \mathrm{CI}=$ $0.878-0.899, p=.005)$.

Regression analysis identified a significantly higher risk of severe hypocalcemia at a combined $\mathrm{pRBC}+\mathrm{FFP}$ administration of 4 units (AOR 18.706, AUC.897 (0.884-0.909) (Fig. 1). Covariates included ISS, penetrating trauma and platelet*pRBC variables.

\section{Discussion}

Transfusion of pRBC was an independent predictors of severe hypocalcemia and ionized calcium decreased in a dose-dependent relationship with transfusion of pRBC. Total calcium is often used as a surrogate for ionized calcium, adjusting for hypoalbuminemia. There are several studies that suggest that the adjusted calcium formula does not adequately reflect ionized calcium concentrations and is not a reliable substitute in critically ill trauma patients [2-6] In our study, ionized calcium values were utilized exclusively. The incidence of hypocalcemia in the adult critically ill trauma population ranges widely from 55 to $97 \%$ with severe hypocalcemia rates of $6.2-71 \%$ depending on the definition of hypocalcemia used $[2,14]$. Our lower hypocalcemia rate of $9.8 \%$ reflects the strict definition used as well as the inclusion of patients beyond the massively transfused patient population. To our knowledge, this is the largest study to date examining the relationship of blood administration to hypocalcemia.

Livingston et al. found no difference in hypocalcemia rates (41 vs $46 \%, p=1.0$ ) when comparing massively 
Table 1 Demographics of Trauma Patients with Severe Hypocalcemia compared to No Severe Hypocalcemia from January 2004 to December $2014(n=7341)$

\begin{tabular}{|c|c|c|c|c|}
\hline Variable & Total, $n=7341$ & $\begin{array}{l}\text { Severe hypocalcemia, } \\
n=716\end{array}$ & $\begin{array}{l}\text { No severe hypocalcemia, } \\
n=6625\end{array}$ & $P$ value \\
\hline Age, years, median (IQR) & $39.0(26-55)$ & $33.0(24-50)$ & $40.0(26-56)$ & $<0.0001$ \\
\hline Male gender, $n(\%)$ & $5960(81.2)$ & $581(81.1)$ & $5379(81.2)$ & 0.9690 \\
\hline Race, $n(\%)$ & 7341 & 716 & 6625 & $<0.0001$ \\
\hline Hispanic & $4182(57.0)$ & $429(59.9)$ & $3753(56.6)$ & \\
\hline White & $1596(21.7)$ & $123(17.2)$ & $1473(22.2)$ & \\
\hline Asian & $725(9.9)$ & $73(10.2)$ & $652(9.8)$ & \\
\hline Black & $597(8.1)$ & $71(9.9)$ & $526(7.9)$ & \\
\hline Other & $194(2.6)$ & $17(2.4)$ & $177(2.7)$ & \\
\hline Unknown & $47(0.6)$ & $3(0.4)$ & $44(0.7)$ & \\
\hline Weight, $\mathrm{kg}$, mean $\pm \mathrm{SD}$ & $77.1 \pm 11.8$ & $76.0 \pm 9.8$ & $77.1 \pm 12$ & 0.8320 \\
\hline Mechanism, $n(\%)$ & 7341 & 716 & 6625 & $<0.0001$ \\
\hline Blunt & $4966(67.6)$ & $367(51.3)$ & $4599(69.4)$ & \\
\hline Fall & $1484(20.2)$ & $53(7.4)$ & $1431(21.6)$ & \\
\hline MVC & $1391(18.9)$ & $100(14.0)$ & $1291(19.5)$ & \\
\hline AVP/AVB & $1121(15.3)$ & $139(19.4)$ & $982(14.8)$ & \\
\hline Assault & $423(5.8)$ & $18(2.5)$ & $405(6.1)$ & \\
\hline $\mathrm{MCC}$ & $384(5.2)$ & $42(5.9)$ & $342(5.2)$ & \\
\hline Fall $>15 \mathrm{ft}$ & $163(2.2)$ & $15(2.1)$ & $148(2.2)$ & \\
\hline Penetrating & $1740(23.7)$ & $312(43.6)$ & $1428(21.6)$ & \\
\hline GSW & $1132(15.4)$ & $224(31.3)$ & 908 (13.7) & \\
\hline SW & $608(8.3)$ & $88(12.3)$ & $520(7.8)$ & \\
\hline Other & $435(5.9)$ & $27(3.8)$ & $408(6.2)$ & \\
\hline Unknown & $200(2.7)$ & $10(1.4)$ & $190(2.9)$ & \\
\hline ISS, median (IQR) & $14(9-25)$ & $25(14-34)$ & $14(9-22)$ & $<0.0001$ \\
\hline Admission GCS, median (IQR) & $15(10-15)$ & $13(3-15)$ & $15(11-15)$ & $<0.0001$ \\
\hline $\mathrm{GCS} \leq 8, n(\%)$ & $1525(20.8)$ & $277(38.7)$ & $1248(18.8)$ & $<0.0001$ \\
\hline Admission SBP, median (IQR) & $131(114-150)$ & $118(90-138)$ & $133(115-151)$ & $<0.0001$ \\
\hline Hypotension, SBP $<90, n(\%)$ & $501(6.8)$ & $166(23.2)$ & $335(5.1)$ & $<0.0001$ \\
\hline Admission HR, median (IQR) & $95(80-113)$ & $106(82-129)$ & $95(80-112)$ & $<0.0001$ \\
\hline Tachycardia, HR $\geq 100, n(\%)$ & $3186(43.4)$ & $408(57.0)$ & $2778(41.9)$ & $<0.0001$ \\
\hline Maximum AIS head, median (IQR) & $0(0-3)$ & $0(0-4)$ & $0(0-3)$ & 0.7960 \\
\hline Maximum AIS face, median (IQR) & $0(0-0)$ & $0(0-0)$ & $0(0-0)$ & 0.2290 \\
\hline Maximum AIS chest, median (IQR) & $0(0-3)$ & $2(0-3)$ & $0(0-2)$ & $<0.0001$ \\
\hline Maximum AIS abdomen/pelvis, median (IQR) & $0(0-2)$ & $(0-3)$ & $(0-2)$ & $<0.0001$ \\
\hline Maximum AIS extremities, median (IQR) & $0(0-3)$ & $(0-3)$ & $(0-3)$ & 0.0180 \\
\hline Maximum AIS external, median (IQR) & $1(0-1)$ & $(0-1)$ & $(0-1)$ & $<0.0001$ \\
\hline
\end{tabular}

Severe hypocalcemia defined as ionized calcium less than $0.9 \mathrm{mmol} / \mathrm{dL}$ or $3.6 \mathrm{mg} / \mathrm{dL}$ within $48 \mathrm{~h}$ of admission

$M V C$ motor vehicle collision, $A V P$ automobile versus pedestrian, $A V B$ automobile versus bicycle, $M C C$ motorcycle collision, $G S W$ gunshot wound, $S W$ stab wound, ISS injury severity score, GCS glasgow coma scale, $S B P$ systolic blood pressure, $H R$ heart rate, $A I S$ abbreviated injury scale

transfused $(\geq 40 \mathrm{~mL} / \mathrm{kg})$ to non-massively transfused $(<40 \mathrm{~mL} / \mathrm{kg})$ pediatric patients. Hypocalcemia was defined as either serum calcium $<2.15 \mathrm{mmol} / \mathrm{L}$ (with correction by albumin when possible) or serum ionized calcium $<0.9 \mathrm{mmol} / \mathrm{L}$. In this smaller study, of the 13 patients that received massive transfusion, only eight received plasma and platelet in the first $24 \mathrm{~h}$, which is different from the balanced resuscitation principles being utilized today [15]. Giancarelli et al. noted a high incidence of hypocalcemia, defined as $\mathrm{iCa}<1.12 \mathrm{mmol}$ or $\mathrm{iCa}<$ 
Table 2 Interventions of trauma patients with severe hypocalcemia compared to no severe hypocalcemia from January 2004 to December 2014 $(n=7341)$

\begin{tabular}{|c|c|c|c|c|}
\hline Variable & Total, $n=7341$ & $\begin{array}{l}\text { Severe hypocalcemia, } \\
n=716\end{array}$ & $\begin{array}{l}\text { No severe hypocalcemia, } \\
n=6625\end{array}$ & $P$ value \\
\hline Intubated, $n(\%)$ & $2211(30.3)$ & $337(47.1)$ & $1874(28.4)$ & $<0.0001$ \\
\hline Intravenous fluids, median (IQR) & $1000(860-2000)$ & $1000(1000-2000)$ & $1000(800-2000)$ & 0.0420 \\
\hline Next Phase After ED, $n(\%)$ & 7341 & 716 & 6625 & $<0.0001$ \\
\hline ICU & $2899(39.5)$ & $160(22.3)$ & $2739(41.3)$ & \\
\hline OR & $2432(33.1)$ & $514(71.8)$ & $1918(29.0)$ & \\
\hline Ward & $1799(24.5)$ & $34(4.7)$ & 1765 (26.6) & \\
\hline Stepdown & $107(1.5)$ & $2(0.3)$ & $105(1.6)$ & \\
\hline Unknown & $89(1.2)$ & $4(0.6)$ & $85(1.3)$ & \\
\hline Other & $15(0.2)$ & $2(0.3)$ & $13(0.2)$ & \\
\hline \multicolumn{5}{|l|}{ pRBC, unit, median (IQR) } \\
\hline Prior to $\min \mathrm{iCa}$ & $0(0-2)$ & $8(4-15)$ & $0(0-2)$ & $<0.0001$ \\
\hline Total, first $24 \mathrm{~h}$ & $0(0-3)$ & $11(6-21)$ & $0(0-2)$ & $<0.0001$ \\
\hline Total, length of stay & $1(0-4)$ & $14(8-25)$ & $0(0-4)$ & $<0.0001$ \\
\hline \multicolumn{5}{|l|}{ FFP, unit, median (IQR) } \\
\hline Prior to min $\mathrm{iCa}$ & $0(0-0)$ & $4(2-8)$ & $0(0-0)$ & $<0.0001$ \\
\hline Total, first $24 \mathrm{~h}$ & $0(0-2)$ & $8(4-14)$ & $0(0-0)$ & $<0.0001$ \\
\hline Total, length of stay & $0(0-2)$ & $9(4-18)$ & $0(0-2)$ & $<0.0001$ \\
\hline \multicolumn{5}{|l|}{ Platelet, unit, median (IQR) } \\
\hline Prior to $\min \mathrm{iCa}$ & $0(0-0)$ & $0(0-2)$ & $0(0-0)$ & $<0.0001$ \\
\hline Total, first $24 \mathrm{~h}$ & $0(0-0)$ & $1(0-3)$ & $0(0-0)$ & $<0.0001$ \\
\hline Total, length of stay & $0(0-0)$ & $2(0-4)$ & $0(0-2)$ & $<0.0001$ \\
\hline \multicolumn{5}{|l|}{ Cryoprecipitate, unit, median (IQR) } \\
\hline Prior to $\min \mathrm{iCa}$ & $0(0-0)$ & $0(0-0)$ & $0(0-0)$ & $<0.0001$ \\
\hline Total, first $24 \mathrm{~h}$ & $0(0-0)$ & $0(0-0)$ & $0(0-0)$ & $<0.0001$ \\
\hline Total, length of stay & $0(0-0)$ & $0(0-0)$ & $0(0-0)$ & $<0.0001$ \\
\hline Transfusion given in first $24 \mathrm{~h}, n(\%)$ & $2849(38.8)$ & $678(94.7)$ & 2178 (32.9) & $<0.0001$ \\
\hline Factor VII given in first $24 \mathrm{~h}, n(\%)$ & $153(2.1)$ & $104(14.5)$ & $49(0.7)$ & $<0.0001$ \\
\hline Massive transfusion patient, $n(\%)$ & $667(9.1)$ & $425(59.4)$ & $242(3.7)$ & $<0.0001$ \\
\hline First iCa, median (IQR) & $4(3.9-4.6)$ & $4(3.4-4.4)$ & $4.4(4.0-4.7)$ & $<0.0001$ \\
\hline Minimum iCa first $48 \mathrm{~h}$, median (IQR) & $4.4(4.0-4.6)$ & $3.1(2.5-3.4)$ & $4.4(4.1-4.7)$ & $<0.0001$ \\
\hline Maximum iCa in first $48 \mathrm{~h}$, median (IQR) & $4.8(4.6-5.0)$ & $4.8(4.5-5.1)$ & $4.8(4.6-4.9)$ & 0.3760 \\
\hline Last iCa in first $48 \mathrm{~h}$, median (IQR) & $4.7(4.4-4.8)$ & $4.5(4.0-4.8)$ & $4.7(4.5-4.8)$ & $<0.0001$ \\
\hline Time to min iCa, minutes, median (IQR) & $338.0(149-955.5)$ & $185.0(108-339)$ & $408.5(169-1114)$ & \\
\hline Time to max iCa, minutes, median (IQR) & $\begin{array}{l}706.0 \\
\quad(224-1743.5)\end{array}$ & $453.0(154 .-1293)$ & $776.5(249-1815.5)$ & $<0.0001$ \\
\hline Intervention in $48 \mathrm{~h}, n(\%)$ & 7341 & 716 & 6625 & $<0.0001$ \\
\hline None & $5117(69.7)$ & $298(41.6)$ & 4819 (72.7) & \\
\hline OR & $2195(29.9)$ & $408(57.0)$ & $1787(27.0)$ & \\
\hline IR & $16(0.2)$ & $2(0.3)$ & $14(0.2)$ & \\
\hline Both & $13(0.2)$ & $8(1.1)$ & $5(0.1)$ & \\
\hline
\end{tabular}

Severe hypocalcemia defined as ionized calcium less than $0.9 \mathrm{mmol} / \mathrm{dL}$ or $3.6 \mathrm{mg} / \mathrm{dL}$ within $48 \mathrm{~h}$ of admission

Massive transfusion defined as 10 unit pRBC or more in $24 \mathrm{~h}$

$E D$ Emergency Department, $I C U$ intensive care unit, $O R$ operating room, $p P R B C$ packed red blood cells, $\min$ minimum, $i C a$ ionized calcium, $F F P$ fresh frozen plasma, max maximum, $I R$ interventional radiology 
Table 3 Outcomes of trauma patients with severe hypocalcemia compared to no severe hypocalcemia from January 2004 to December 2014 $(n=7341)$

\begin{tabular}{llll}
\hline Variable & $\begin{array}{l}\text { Total, } \\
n=7341\end{array}$ & $\begin{array}{l}\text { Severe hypocalcemia, } \\
n=716\end{array}$ & $\begin{array}{l}\text { No severe hypocalcemia, } \\
n=6625\end{array}$ \\
\hline Discharge capacity, $n(\%)$ & 7341 & 716 & 6625 \\
Temporary disability, $<1$ year & $6119(83.4)$ & $410(57.3)$ & $5709(86.2)$ \\
Deceased or unknown & $990(13.5)$ & $273(38.1)$ & $717(10.8)$ \\
Disabled & $204(2.8)$ & $31(4.3)$ & $173(2.6)$ \\
Pre-injury capacity & $28(0.4)$ & $2(0.3)$ & $26(0.4)$ \\
Discharge disposition, $n(\%)$ & 7341 & 716 & 6625 \\
Home & $3835(52.2)$ & $248(34.6)$ & $3587(54.1)$ \\
Transfer to OSH & $1106(15.1)$ & $92(12.8)$ & $1014(15.3)$ \\
Expired & $932(12.7)$ & $270(37.7)$ & $662(10.0)$ \\
SNF/rehab & $783(10.7)$ & $62(8.7)$ & $721(10.9)$ \\
Other & $685(9.3)$ & $44(6.1)$ & $641(9.7)$ \\
Required ICU, $n(\%)$ & $5486(74.7)$ & $571(79.7)$ & $4915(74.2)$ \\
Hospital LOS, median (IQR) & $7(4-17)$ & $9(1-26)$ & $7(4-16)$ \\
Ventilator days, median (IQR) & $0(0-2)$ & $0(0-4)$ & $0(0-2)$ \\
ICU LOS, median (IQR) & $3(0-8)$ & $4(1-14)$ & $3(0-7)$ \\
In-hospital mortality, $n(\%)$ & $936(12.8)$ & $270(37.7)$ & $666(10.1)$ \\
\hline Sever & & & 0.0001 \\
\end{tabular}

Severe hypocalcemia defined as ionized calcium less than $0.9 \mathrm{mmol} / \mathrm{dL}$ or $3.6 \mathrm{mg} / \mathrm{dL}$ within $48 \mathrm{~h}$ of admission

Disabled defined as limitations from injuries expected to last longer than 1 year

$O S H$ outside hospital, SNF skilled nursing facility, rehab rehabilitation facility, ICU intensive care unit, LOS length of stay

Table 4 Multivariable analysis regarding the association between variables and severe hypocalcemia

\begin{tabular}{llll}
\hline Variables & \multicolumn{3}{c}{ Clinically significant severe hypocalcemia } \\
\cline { 2 - 4 } & Adjusted $p$ value & AOR & CI \\
\hline Penetrating & $<0.0001$ & 1.706 & $1.383,2.103$ \\
ISS & $<0.0001$ & 1.029 & $1.021,1.037$ \\
PRBC prior to iCa nadir & $<0.0001$ & 1.343 & $1.306,1.381$ \\
FFP prior to iCa nadir & $<0.0001$ & 1.097 & $1.057,1.14$ \\
Plt prior to iCa nadir & 0.218 & & \\
PRBC Plt & $<0.0001$ & 0.971 & $0.966,0.977$ \\
Cryo prior to iCa nadir & 0.126 & & \\
Age & 0.960 & & \\
\hline
\end{tabular}

Severe hypocalcemia defined as ionized calcium less than $0.9 \mathrm{mmol} /$ $\mathrm{dL}$ or $3.6 \mathrm{mg} / \mathrm{dL}$ within $48 \mathrm{~h}$ of admission. Transfused blood products were maintained as continuous variables in the regression model.

${ }^{a}$ Backward stepwise logistical regression performed with variables which were significant to $p \leq 0.2$ in the univariate analysis. Significant variables were then included in the final logistical model using "enter" mode

AUROC $=0.909(95 \%$ CI $=0.897-0.921, p<0.0001)$

ISS injury severity score, $P R B C$ packed red blood cells, $i C a$ ionized calcium, FFP fresh Frozen plasma, Plt platelet, Cryo cryoprecipitate
$4.48 \mathrm{mg} / \mathrm{dL}$, with $152(97 \%)$ of 156 trauma patients receiving a massive transfusion demonstrating hypocalcemia. Severe hypocalcemia $(\mathrm{iCa}<0.9 \mathrm{mmol} / \mathrm{L}$ or $\mathrm{iCa}<3.6 \mathrm{mg} / \mathrm{dL}$ ) was seen in $71 \%$ in this massive transfusion population [14].

Previously, Kraft et al. [16] established a relationship between the amount of pRBC administered and hypocalcemia. Patients with severe hypocalcemia had more units (34 vs 22) of pRBC transfused compared to patients without severe hypocalcemia in a retrospective analysis of 156 patients by Giancarelli et al. [14]. ROC analysis in the massive transfusion patient cohort suggested that the transfusion of 15 units of total blood products was the best predictor for severe hypocalcemia. In our study, which expanded the population to all trauma patients that had an ionized calcium drawn, we found 4 units total of PRBC and FFP was associated with severe hypocalcemia. Since plasma can be administered rapidly due to its low viscosity, and has the highest concentration of citrate per unit volume of any blood product, ionized calcium is most likely to occur during FFP infusion [17]. Furthermore, FFP and platelet contain $>90 \%$ of the citrate contained in the original blood donation bag [8]. Our results are consistent with a dose-response relationship between the volume of blood product administered and severity of hypocalcemia. 


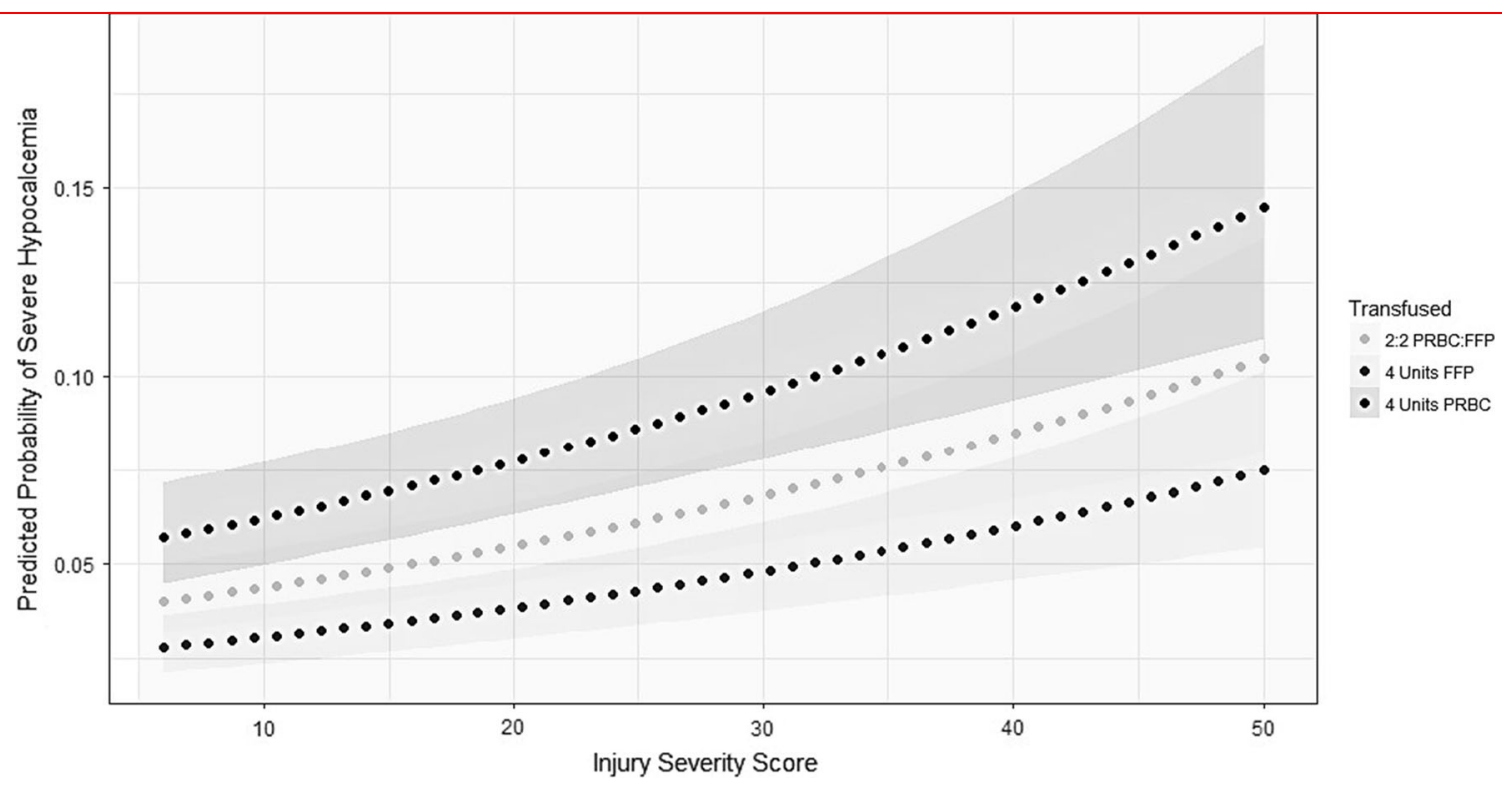

Fig. 1 Adjusted for covariates, the probability of developing severe hypocalcemia increases with transfusion of PRBC, FFP, or both. Covariates ISS, penetrating mechanism, PRBC X Plt. Abbreviations: ISS-Injury Severity Score, PRBC-Packed Red Blood Cells, FFP-Fresh Frozen Plasma, Plt-Platelets. The gray bands are $95 \%$ confidence intervals

Poor clinical outcomes have been associated with hypocalcemia [6, 18-23], and low admission ionized calcium is an independent predictor of multiple transfusions [23]. Furthermore, in several large studies, severe hypocalcemia is independently associated with increased mortality. Inability to normalize ionized calcium by day 4 has been associated with a doubling of mortality [2]. In this study, severe hypocalcemia was also found to be an independent predictor of mortality.

Previous studies suggest that calcium replacement may not improve calcium normalization or mortality [2]. Calcium replacement protocols vary in the literature, and the role of replacement is uncertain. Cote et al. prospectively evaluated pediatric burn patients that required FFP infusion, and concluded that concurrent calcium replacement was potentially indicated to avoid dangerous fluctuations in ionized calcium. The study was originally undertaken after a number of cardiac arrests, and severe hypotensive episodes were witnessed in association with FFP infusion. Another pediatric study by Krishnan et al. examined calcium and magnesium supplementation during plasmapheresis and FFP transfusion in children with glomerulonephritis. The concentration of ionized calcium fell by similar proportions during FFP infusion in both the supplemented and standard cohorts but with higher measured values of absolute ionized calcium $(0.92 \mathrm{mmol} / \mathrm{L}$ compared to $0.78 \mathrm{mmol} / \mathrm{L}$ ) and decreased clinical symptoms of tetany (0\% vs $29 \%$ ) in the supplemented group [24]. They subsequently protocolized routine calcium and magnesium supplementation and eliminated hypocalcemia symptoms from their population. An adult population of 84 patients that underwent 581 plasma exchanges for various autoimmune indications reported a lower incidence of tetany, which they partially attributed to their routine calcium supplementation as well as slower citrate infusion rates [25]. Although we did not examine the role of calcium supplementation in our study, this is an area that warrants further investigation in the trauma population. In this study, administration of pRBC and FFP was independent predictors of hypocalcemia and a total of 4 units pRBC + FFP was associated with severe hypocalcemia. Furthermore, the predicted probability of severe hypocalcemia increased as the total $\mathrm{pRBC}+\mathrm{FFP}$ administered increased.

\section{Conclusion}

Transfusion of pRBC and FFP are independent predictors of severe hypocalcemia, as the value of $\mathrm{pRBC}+\mathrm{FFP}$ administered increase, the predicted probability of severe hypocalcemia also increases. A total of 4 units pRBC + FFP is associated with significantly higher risk of severe hypocalcemia.

Author contribution Conception, design, overall responsibility: KI. Data collection: SB, EW, MW. Statistical analysis: SB, SB. Analysis and interpretation: SB, SB, EW, KI. Writing the article: SB. Critical 
revision of the article: KI, SB, SB, MW, EB, LL, DD. Final approval of the article: KI.

\section{Compliance with ethical standards}

Conflict of interest We have no conflicts of interest and no sources of funding to disclose. IRB approval was obtained and informed consent was waived in compliance with institutional and national guidelines.

\section{References}

1. Zaloga GP, Chernow B (1986) Hypocalcemia in critical illness. JAMA 256:1924-1929

2. Steele T, Kolamunnage-Dona R, Downey C et al (2013) Assessment and clinical course of hypocalcemia in critical illness. Crit Care 17:R106

3. Slomp J, van der Voort PHJ, Gerritsen RT et al (2003) Albuminadjusted calcium is not suitable for diagnosis of hyper- and hypocalcemia in the critically ill. Crit Care Med 31:1389-1393

4. Dickerson RN, Alexander KH, Minard G et al (2004) Accuracy of methods to estimate ionized and 'corrected' serum calcium concentrations in critically ill multiple trauma patients receiving specialized nutrition support. JPEN J Parenter Enteral Nutr 28:133-141

5. Byrnes MC, Huynh K, Helmer SD et al (2005) A comparison of corrected serum calcium levels to ionized calcium levels among critically ill surgical patients. Am J Surg 189:310-314

6. Chernow B, Zaloga G, McFadden E et al (1982) Hypocalcemia in critically ill patients. Crit Care Med 10:848-851

7. Brody T (1998) Nutritional biochemistry, 2nd edn. Academic Press, San Diego

8. Sheridan R (2004) The trauma handbook of the Massachusetts general hospital, 1st edn. Lippincott Williams \& Wilkins, Boston

9. (1988) Guidelines for transfusion for massive blood loss. A publication of the British Society for Haematology. British Committee for Standardization in Haematology Blood Transfusion Task Force. Clin Lab Haematol 10: 265-273

10. Perkins JG, Cap AP, Weiss BM et al (2008) Massive transfusion and nonsurgical hemostatic agents. Crit Care Med 36:S325-S339

11. Kramer L, Bauer E, Joukhadar C et al (2003) Citrate pharmacokinetics and metabolism in cirrhotic and noncirrhotic critically ill patients. Crit Care Med 31:2450-2455

12. Ludbrook J, Wynn V (1958) Citrate intoxication; a clinical and experimental study. Br Med J 2:523-528
13. Bunker JP, Bendixen HH, Murphy AJ (1962) Hemodynamic effects of intravenously administered sodium citrate. N Engl J Med 266:372-377

14. Giancarelli A, Birrer KL, Alban RF et al (2016) Hypocalcemia in trauma patients receiving massive transfusion. J Surg Res 202:182-187

15. Livingston MH, Singh S, Merritt NH (2014) Massive transfusion in paediatric and adolescent trauma patients: incidence, patient profile, and outcomes prior to a massive transfusion protocol. Injury 45:1301-1306

16. Kraft MD, Btaiche IF, Sacks GS et al (2005) Treatment of electrolyte disorders in adult patients in the intensive care unit. Am J Health Syst Pharm 62:1663-1682

17. Cote CJ, Drop LJ, Hoaglin DC et al (1988) Ionized hypocalcemia after fresh frozen plasma administration to thermally injured children: effects of infusion rate, duration, and treatment with calcium chloride. Anesth Analg 67:152-160

18. Desai TK, Carlson RW, Geheb MA (1988) Prevalence and clinical implications of hypocalcemia in acutely ill patients in a medical intensive care setting. Am J Med 84:209-214

19. Desai TK, Carlson RW, Thill-Baharozian M et al (1988) A direct relationship between ionized calcium and arterial pressure among patients in an intensive care unit. Crit Care Med 16:578-582

20. Burchard KW, Gann DS, Colliton J et al (1990) Ionized calcium, parathormone, and mortality in critically ill surgical patients. Ann Surg 212:543-550

21. Carlstedt F, Lind L, Rastad J et al (1998) Parathyroid hormone and ionized calcium levels are related to the severity of illness and survival in critically ill patients. Eur J Clin Invest 28:898-903

22. Cherry RA, Bradburn E, Carney DE et al (2006) Do early ionized calcium levels really matter in trauma patients? J Trauma 61:774-779

23. Magnotti LJ, Bradburn EH, Webb DL et al (2011) Admission ionized calcium levels predict the need for multiple transfusions: a prospective study of 591 critically ill trauma patients. J Trauma 70:391-397

24. Krishnan RG, Coulthard MG (2007) Minimising changes in plasma calcium and magnesium concentrations during plasmapheresis. Pediatr Nephrol 22:1763-1766

25. Kankirawatana S, Huang ST, Marques MB (2007) Continuous infusion of calcium gluconate in 5\% albumin is safe and prevents most hypocalcemic reactions during therapeutic plasma exchange. J Clin Apher 22:265-269

Publisher's Note Springer Nature remains neutral with regard to jurisdictional claims in published maps and institutional affiliations. 